\title{
Intersecting Line of Conical Surface and Smoothly Blending of Two Tubes Whose Axes Are Non-Coplanar
}

\author{
Genzhu Bai ${ }^{1,2}$, Zhi Wu1, Xiao Lin'1 \\ ${ }^{1}$ School of Mathematics, Inner Mongolia University for the Nationalities, Tongliao, China \\ ${ }^{2}$ Zhe Jiang Chang Zheng Vocational \& Technical College, Hangzhou, China \\ Email: bgz049@163.com
}

How to cite this paper: Bai, G.Z., Wu, Z. and Lin, $X$. (2017) Intersecting Line of Conical Surface and Smoothly Blending of Two Tubes Whose Axes Are Non-Coplanar. Journal of Applied Mathematics and Physics, 5, 1887-1891.

https://doi.org/10.4236/jamp.2017.59158

Received: September 12, 2017

Accepted: October 13, 2017

Published: October 16, 2017

\begin{abstract}
We construct two conical surfaces which take non-coplanar lines as generatrix and rational Bezier curve as ridge-line, and prove that the intersecting line of conical surface has similar properties to Bezier curve. Then, the smoothly blending of two cylinders whose axes are non-coplanar is realized by taking intersecting line of conical surface as axes.
\end{abstract}

\section{Keywords}

Bezier Curve, Intersecting Line of Conical Surface, Tubes, Non-Coplanar Tubes, Smoothly Blending

\section{Introduction}

Blending surface is one of the main research fields of curve and surface modeling technology. In recent years, many scholars have focused on algebraic surface blending [1]-[5], namely of Grobner based method, Wu method, and Resultant method that is to study implicit surface blending. The common feature of these studies is that they are all coplanar algebraic surface smooth blending. Ideal blending surfaces is obtained in theory, but the shape of the transition surface is unknown when the algebraic surface is blended. Moreover, there is a certain difference between the actual surface designing the demand and the blending surface. In addition, some tubes are crossing in the industrial manufacturing process because of the obstacle blocking factors or other reasons. Not only do we need to consider the parameter surface, but also the shape of the smooth surface. Therefore, it is necessary to consider the study of the smoothly blending of two cylinders whose axes are non-coplanar. The main purpose of the method is to 
manufacture the special process functions in the industry. In the [6] [7] [8] [9], the tubes with a helix as the axis have blended smoothly between circular tubes and elliptical tubes. Furthermore, in the [10] [11], tubes with intersecting line of conical surface as the axis are blended smoothly between special circular tubes. In this paper, a more general case is discussed.

\section{Structuring Ridge-Line of Conical Surface and Intersecting Line of Conical Surface}

In this paper, we construct equation of ridge-line of conical surface, and blend smoothly two elliptical tubes whose axes are non-coplanar with the elliptical tube that takes intersecting line of conical surface as axes. In the case of dihedral angle of axes line is 90 degree, more than 90 , and less than 90 , the tube that takes intersecting line of conical surface as axes blend smoothly the two tubes whose axes is non-coplanar. Let $l_{1}$ and $l_{2}$ be non-coplanar straight line, $a$ is the shortest distance between the two lines, select the $x$ axis so that it is parallel to the shortest distance segment between $l_{1}$ and the $l_{2}$, the $y$ axis is parallel to $l_{1}$, and intersects the $l_{2}$. Establishing right hand Cartesian coordinate system, so that the intersection point of $l_{1}$ and $x$ axis is point $V_{0}(a, 0,0)$, and the intersection point of $y$ axis and $l_{2}$ is point $V_{2}(0, a, 0)$, the point $V_{0}(a, 0,0)$ and $V_{3}(0, c, d)$ on $l_{2}$ forms four points, these point are no coplanar. Respectively, with $V_{0}(a, 0,0)$ and $V_{3}(0, c, d)$ as the cone-vertices of the conical surface, and $V_{1}(a, a, 0), V_{2}(0, a, 0), V_{3}(0, c, d)$, and $V_{0}(a, a, 0), V_{1}(0, a, 0), V_{2}(0, c, d)$ are the vertices of the characteristic polygons. $m_{1}, m_{2}, m_{3}$ and $w_{1}, w_{2}, w_{3}$ are the corresponding the vertex of weight. The parameter equation for ridge-line of conical surface and equation of conical surface are as follows.

$$
\begin{gathered}
\left\{\begin{array}{l}
x(u)=\frac{m_{1} \cdot a \cdot(1-u)^{2}+2 m_{2} \cdot 0 \cdot u(1-u)+m_{3} \cdot 0 \cdot u^{2}}{m_{1} \cdot(1-u)^{2}+2 m_{2} \cdot u(1-u)+m_{3} \cdot u^{2}}, \\
y(u)=\frac{m_{1} \cdot a \cdot(1-u)^{2}+2 m_{2} \cdot a \cdot u(1-u)+m_{3} \cdot c \cdot u^{2}}{m_{1} \cdot(1-u)^{2}+2 m_{2} \cdot u(1-u)+m_{3} \cdot u^{2}}, \\
z(u)=\frac{m_{1} \cdot 0 \cdot(1-u)^{2}+2 m_{2} \cdot 0 \cdot u(1-u)+m_{3} \cdot d \cdot u^{2}}{m_{1} \cdot(1-u)^{2}+2 m_{2} \cdot u(1-u)+m_{3} \cdot u^{2}} .
\end{array}\right. \\
\left\{\begin{array}{l}
x(s)=\frac{w_{1} \cdot a \cdot(1-s)^{2}+2 w_{2} \cdot a \cdot s(1-s)+w_{3} \cdot 0 \cdot s^{2}}{w_{1} \cdot(1-s)^{2}+2 w_{2} \cdot s(1-s)+w_{3} \cdot s^{2}}, \\
y(s)=\frac{w_{1} \cdot 0 \cdot(1-s)^{2}+2 w_{2} \cdot a \cdot s(1-s)+w_{3} \cdot a \cdot u^{2}}{w_{1} \cdot(1-s)^{2}+2 w_{2} \cdot s(1-s)+w_{3} \cdot s^{2}} \\
z(s)=\frac{w_{1} \cdot 0 \cdot(1-s)^{2}+2 w_{2} \cdot 0 \cdot s(1-s)+w_{3} \cdot 0 \cdot s^{2}}{w_{1} \cdot(1-s)^{2}+2 w_{2} \cdot s(1-s)+w_{3} \cdot s^{2}} . \\
x(u, v)=a+\left(\frac{m_{1} \cdot a \cdot(1-u)^{2}+2 m_{2} \cdot 0 \cdot u(1-u)+m_{3} \cdot 0 \cdot u^{2}}{m_{1} \cdot(1-u)^{2}+2 m_{2} \cdot u(1-u)+m_{3} \cdot u^{2}}-a\right) v, \\
y(u, v)=0+\left(\frac{m_{1} \cdot a \cdot(1-u)^{2}+2 m_{2} \cdot a \cdot u(1-u)+m_{3} \cdot c \cdot u^{2}}{m_{1} \cdot(1-u)^{2}+2 m_{2} \cdot u(1-u)+m_{3} \cdot u^{2}}-0\right) v, \\
z(u, v)=0+\left(\frac{m_{1} \cdot 0 \cdot(1-u)^{2}+2 m_{2} \cdot 0 \cdot u(1-u)+m_{3} \cdot d \cdot u^{2}}{m_{1} \cdot(1-u)^{2}+2 m_{2} \cdot u(1-u)+m_{3} \cdot u^{2}}-0\right) v .
\end{array}\right.
\end{gathered}
$$




$$
\begin{aligned}
& \left\{\begin{array}{l}
x(s, t)=0+\left(\frac{w_{1} \cdot a \cdot(1-s)^{2}+2 w_{2} \cdot a \cdot s(1-s)+w_{3} \cdot 0 \cdot s^{2}}{w_{1} \cdot(1-s)^{2}+2 w_{2} \cdot s(1-s)+w_{3} \cdot s^{2}}-0\right) t, \\
y(u, v)=c+\left(\frac{w_{1} \cdot 0 \cdot(1-s)^{2}+2 w_{2} \cdot a \cdot s(1-s)+w_{3} \cdot a \cdot u^{2}}{w_{1} \cdot(1-s)^{2}+2 w_{2} \cdot s(1-s)+w_{3} \cdot s^{2}}-c\right) t, \\
z(u, v)=d+\left(\frac{w_{1} \cdot 0 \cdot(1-s)^{2}+2 w_{2} \cdot 0 \cdot s(1-s)+w_{3} \cdot 0 \cdot u^{2}}{w_{1} \cdot(1-s)^{2}+2 w_{2} \cdot s(1-s)+w_{3} \cdot s^{2}}-d\right) t .
\end{array}\right. \\
& \int x(s)=-\left(8 w_{2} m_{2}^{2} a(s-1)^{2}\left(w_{1} s-2 w_{2} s-w_{1}\right)\right) /\left(m_{1} m_{3} w_{3}^{2} s^{3}-8 m_{2}^{2} w_{1} w_{2} s^{3}\right. \\
& +16 m_{2}^{2} w_{2}^{2} s^{3}-8 m_{2}^{2} w_{2} w_{3} s^{3}+24 m_{2}^{2} w_{1} w_{2} s^{2}-32 m_{2}^{2} w_{2}^{2} s^{2} \\
& \left.+8 m_{2}^{2} w_{2} w_{3} s^{2}-24 m_{2}^{2} w_{1} w_{2} s+16 m_{2}^{2} w_{2}^{2} s+8 m_{2}^{2} w_{1} w_{2}\right), \\
& y(s)=\left(16 a m_{2}^{2} w_{2}^{2} s^{3}-8 a m_{2}^{2} w_{2} w_{3} s^{3}+c m_{1} m_{3} w_{3}^{2} s^{3}-32 a m_{2}^{2} w_{2}^{2} s^{2}\right. \\
& \left.+8 a m_{2}^{2} w_{2} w_{3} s^{2}+16 a m_{2}^{2} w_{2}^{2} s\right) /\left(m_{1} m_{3} w_{3}^{2} s^{3}-8 m_{2}^{2} w_{1} w_{2} s^{3}\right. \\
& +16 m_{2}^{2} w_{2}^{2} s^{3}-8 m_{2}^{2} w_{2} w_{3} s^{3}+24 m_{2}^{2} w_{1} w_{2} s^{2}-32 m_{2}^{2} w_{2}^{2} s^{2} \\
& \left.+8 m_{2}^{2} w_{2} w_{3} s^{2}-24 m_{2}^{2} w_{1} w_{2} s+16 m_{2}^{2} w_{2}^{2} s+8 m_{2}^{2} w_{1} w_{2}\right), \\
& z(s)=\left(d m_{1} m_{2} w_{3}^{2} s^{3}\right) /\left(m_{1} m_{3} w_{3}^{2} s^{3}-8 m_{2}^{2} w_{1} w_{2} s^{3}+16 m_{2}^{2} w_{2}^{2} s^{3}\right. \\
& -8 m_{2}^{2} w_{2} w_{3} s^{3}+24 m_{2}^{2} w_{1} w_{2} s^{2}-32 m_{2}^{2} w_{2}^{2} s^{2}+8 m_{2}^{2} w_{2} w_{3} s^{2} \\
& \left.-24 m_{2}^{2} w_{1} w_{2} s+16 m_{2}^{2} w_{2}^{2} s+8 m_{2}^{2} w_{1} w_{2}\right) \text {. }
\end{aligned}
$$

Theorem 1. The parameter equations of two conical surfaces are (1) and (2). The parameter equation of the intersection line is

$$
r(s)=(x(s), y(s), z(s)) .
$$

Then the first and end points of the curve have the following properties.

$$
r(0)=V_{0}, r(1)=V_{3} .
$$

The nature of the tangent vector is further introduced.

$$
r^{\prime}(0)=\frac{2 w_{2}}{w_{1}}\left(V_{1}-V_{0}\right), r^{\prime}(1)=\frac{8 m_{2}^{2} w_{2}}{m_{1} m_{2} w_{3}}\left(V_{3}-V_{2}\right) .
$$

Its geometric meaning is that the first and last points of the curve are tangent to the two different lines.

In this way, we can construct a tube with an intersecting line of conical surface as the axis, and smoothly blend the non-coplanar tubes with two non-coplanar lines as axes.

Theorem 2. The parameter equations of non-coplanar lines $l_{1}$ and $l_{2}$ are as follows,

$$
l_{1}:(a, a s, 0) ; l_{2}:(0, c+(c-a) s, d s) .
$$

The curve equation for smoothly blending of two non-coplanar lines is (3), then the parameter equations of two non-coplanar tubes and smoothly blending tube are as follows.

$$
\left\{\begin{array}{l}
x_{1}(s, t)=x_{1}(s)+N_{11}(s) \cos t+B_{11}(s) \sin t \\
y_{1}(s, t)=y_{1}(s)+N_{12}(s) \cos t+B_{12}(s) \sin t \\
z_{1}(s, t)=z_{1}(s)+N_{13}(s) \cos t+B_{13}(s) \sin t
\end{array}\right.
$$




$$
\begin{gathered}
\left\{\begin{array}{l}
x_{2}(s, t)=x_{2}(s)+N_{21}(s) \cos t+B_{21}(s) \sin t, \\
y_{2}(s, t)=y_{2}(s)+N_{22}(s) \cos t+B_{22}(s) \sin t, \\
z_{2}(s, t)=z_{2}(s)+N_{23}(s) \cos t+B_{23}(s) \sin t
\end{array}\right. \\
\left\{\begin{array}{l}
x(s, t)=x(s)+N_{1}(s) \cos t+B_{1}(s) \sin t, \\
y(s, t)=y(s)+N_{2}(s) \cos t+B_{2}(s) \sin t, \\
z(s, t)=z(s)+N_{3}(s) \cos t+B_{3}(s) \sin t
\end{array}\right.
\end{gathered}
$$

where $N=\left(N_{1}, N_{2}, N_{3}\right)$ and $B=\left(B_{1}, B_{2}, B_{3}\right)$ are the unit principal vector and the unit binormal vector at the corresponding point of the intersecting line of conical surface, $N_{i}=\left(N_{i 1}, N_{i 2}, N_{i 3}\right)$ and $B_{i}=\left(B_{i 1}, B_{i 2}, B_{i 3}\right)$, for unit principal vector and unit binormal vector at the intersecting line of conical surface respectively.

\section{Concluding Remarks}

In this paper, by constructing the intersecting line of conical surface in Figure 1, the elliptical tube with the intersecting line of conical surface in Figure 2 as the axis has blended two elliptical tube whose axes are non-coplanar. The blending surfaces of different situations are successfully realized, and the surface meets $G^{1}$ blending conditions. As can be seen from examples, the manufacture of a specific process function is solved in industry.
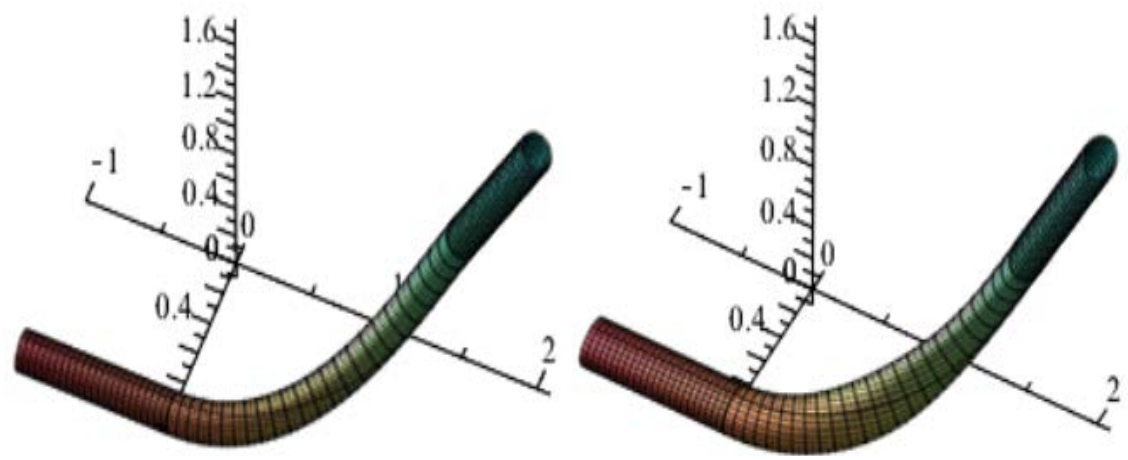

Figure 1. Circular tube blending and elliptical tube blending in the case of dihedral angle of axes line it is greater than 90 degree.

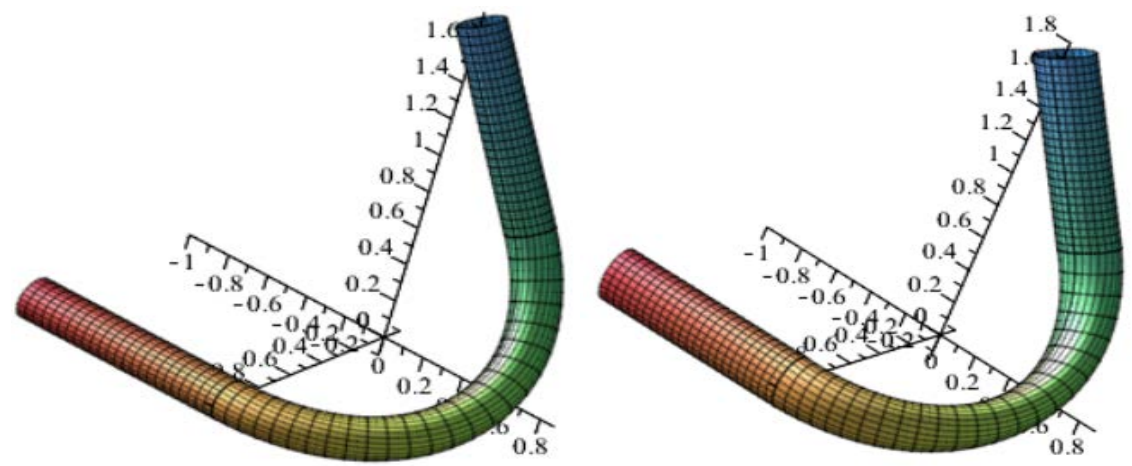

Figure 2. Circular tube blending and elliptical tube blending in the case of dihedral angle of axes line it is less than 90 degree. 


\section{Acknowledgements}

This work is supported by the National Science Fund of China (NSFC11561052) and the Inner Mongolia Natural Science Foundation of China (NMDGP1415).

\section{References}

[1] Warren, J. (1989) Blending Algebraic Surfaces. ACM Transactions on Graphics, 8, 263-278. https://doi.org/10.1145/77269.77270

[2] Wu, W.-T. and Wang, D.-K. (1994) Fitting Problem of Algebraic Surface in CAGD. Journal of Mathematics in Practice and Theory, 3, 26-31.

[3] Bai, G.-Z., Zhan, P.-J. and Lei, N. (2007) Blending of Two Cylinders with Axes in Different Planes. Journal of Jinlin University, 45, 216-218.

[4] Bai, G.-Z. (2006) Blending of Implicit Algebraic Surfaces. Mathematics Practice and Theory, 36, 274-277.

[5] Wu, T.-R. (2005) GC $C^{k}$ Blending Pipe Surface by Ringed Surface for Two Arbitrary Surfaces. Journal of Engineering Graphics, 1, 39-44.

[6] Lei, N., Zhang, S.G., Dong, T. and Feng, G.C. (2005) The Existence and Expression of Osculatory Rational Interpolation. Journal of Information and Computational Science, 2, 493-500.

[7] Bai, G.-Z., Wang, H. and Yin, Z.-J. (2014) Employing Generalized Bezier Tube to Smoothly Blending Tubes Whose Axes Are Non-coplanar. Applied Mechanics and Materials, 513, 2301-2306. https://doi.org/10.4028/www.scientific.net/AMM.513-517.2301

[8] Wang, H. and Bai, G.-Z. (2013) Employing Generalized Cylindrical Helicoid Tube to Smoothly Blending Tubes Whose Axes are Non-coplanar Vehicle. Applied Mechanics and Materials, 380, 380-384.

https://doi.org/10.4028/www.scientific.net/AMM.441.380

[9] Bai, G.-Z. and Wu, Z. (2013) Multi-Degree Reduction Approximation of Bézier Curves by Subdivision method. Intelligent Information Management Systems and Technologies, 9, 37-42.

[10] Bai, G.-Z., Liu, S.-Y., Wang, H., Wu, Z. and Yin, Z.-J. (2014) A Novel Method for Smooth Blending Cylindrical Surfaces Whose Axes Are Non-Coplanar Based on Smooth Blending Axes. Applied Mechanics and Materials, 687, 1470-1473.

[11] Bai, G.-Z., Wang, H., Wu, Z., Yin, Z.-J. and Liu, S.-Y. (2014) Smoothly Blending of Two Elliptic Cylinders Whose Axes Are Non-coplanar. Applied Mechanics and Materials, 687, 644-650.

https://doi.org/10.4028/www.scientific.net/AMM.644-650.309 